\title{
A Further Parametric Study of Imipramine in an Animal Model of Depression
}

\author{
R. J. KATZ AND G. BALDRIGHI \\ Mental Health Research Institute, Department of Psychiatry \\ University of Michigan Medical Center, Ann Arbor, MI 48109
}

Received 16 January 1981

\begin{abstract}
KATZ, R. J. AND G. BALDRIGHI. A further parametric study of imipramine in an animal model of depression. PHARMAC. BIOCHEM. BEHAV. 16(6) 969-972, 1982.-We have proposed that chronic stress may produce motivational, behavioral, and neuroendocrine symptoms in rats resembling endogenous depression in humans. The chronic stress model has proved responsive to chronic treatment by antidepressant drugs. Two issues concerning this effect remain unresolved, these being; the requirement of drug chronicity, and treatment outcome to different drug doses. The present experiment examined both issues in a factorial design in which vehicle and two doses of the tricyclic antidepressant imipramine were varied across 2 treatment periods; acute $(1 \mathrm{hr})$ and chronic $(3$ weeks). Both factors were found to significantly interact with treatment outcome, suggesting that chronic treatment is necessary for recovery and that this outcome is dependent upon drug level.
\end{abstract}

Activity Animal model of depression Antidepressant Defecation Imipramine Open field Stress

IN a recent series of papers (e.g., $[2,3]$ ), we have suggested that chronic stress may produce a behavioral, motivational, and neuroendocrine syndrome in the rat resembling endogenous depression in humans. The syndrome has been demonstrated to respond exclusively to clinically relevant therapeutic treatments. However, two objections might be raised to previous demonstrations. First, the necessity for chronic drug treatment might be questioned: it is possible, for example, that acute drug administration, a treatment with no or even adverse clinical effects [4], would be as effective as chronic treatment. Such an outcome would raise questions concerning the validity of the model. Second, it might be questioned whether treatment outcome depends upon drug dosage level. Since subclinical doses of antidepressants are not normally effective [6] such an outcome again would call the validity of the stress model into question. In the present design both level and chronicity of drug treatment were parametrically varied to allow an assessment of both factors and their interactions.

\section{METHOD}

\section{Subjects}

Adult male Sprague-Dawley rats $(n=144 ; 6 /$ cell, Charles River Farms) were double housed in standard $25 \times 18 \times 17 \mathrm{~cm}$ rack mounted cages with food (Teklad $4.0 \%$ fat rodent diet $\mathrm{S}-0836$ ) and tap water continuously available and $12 \mathrm{hr} / 12 \mathrm{hr}$ lighting (lights on $=0700-1900 \mathrm{hr}$ ).

\section{Apparatus and Behavioral Procedure}

Testing was carried out in a $1.22 \mathrm{~m}^{2} \times 45 \mathrm{~cm}$ height white Plexiglas open field. Each subject was placed in the field for a single 6 minute observation period, with the field cleaned thoroughly between tests. A four-factorial $(2 \times 2 \times 2 \times 3)$ experimental design was employed with the factors being: (1) acute stress vs control (hereafter the acute factor); rats were either removed from housing and tested immediately $(<20$ sec) or removed and stressed with a 1 hour exposure to $95 \mathrm{~dB}$ of white noise prior to the open field test (see [2,3] for further procedural details); (2) chronic stress vs control (hereafter the chronic factor), rats were either exposed to 3 weeks of chronic intermittent stress, with stressors including: exposure to sixty minutes of unpredictable shock (average $1 \mathrm{~mA}$, $1-10 \mathrm{sec}$ duration; average 1 shock/minute ( 3 exposures); 40 hours' food deprivation ( 2 times); cold swim at $4.0^{\circ} \mathrm{C}$ for 5 minutes ( 3 exposures); 40 hours ${ }^{\circ}$ water deprivation ( 2 times); 5 minutes exposure to heat stress at $40^{\circ} \mathrm{C}$ ( 2 times); 30 minutes' shaker stress ( 2 times); reversal of day/night cycle (2 times; stressors were delivered in a semirandom fashion every 2-3 days throughout the day/night cycle from 0:800 through 22:00 hr; order of stress presentation has been presented elsewhere see $[2,3]$; control rats for chronic stress were left undisturbed except for normal cage maintenance (changing of food, water, and bedding) for the 3 week period.); (3) acute vs chronic drug treatment (drug chronicity factor), rats were administered vehicle or drug either 1 hour prior to testing (acute) or on a once daily basis for the 3 week

\footnotetext{
'Send reprint requests to R. J. Katz, Department of Psychology, The Johns Hopkins University, 34th and Charles Streets, Baltimore, MD
} 21218 . 

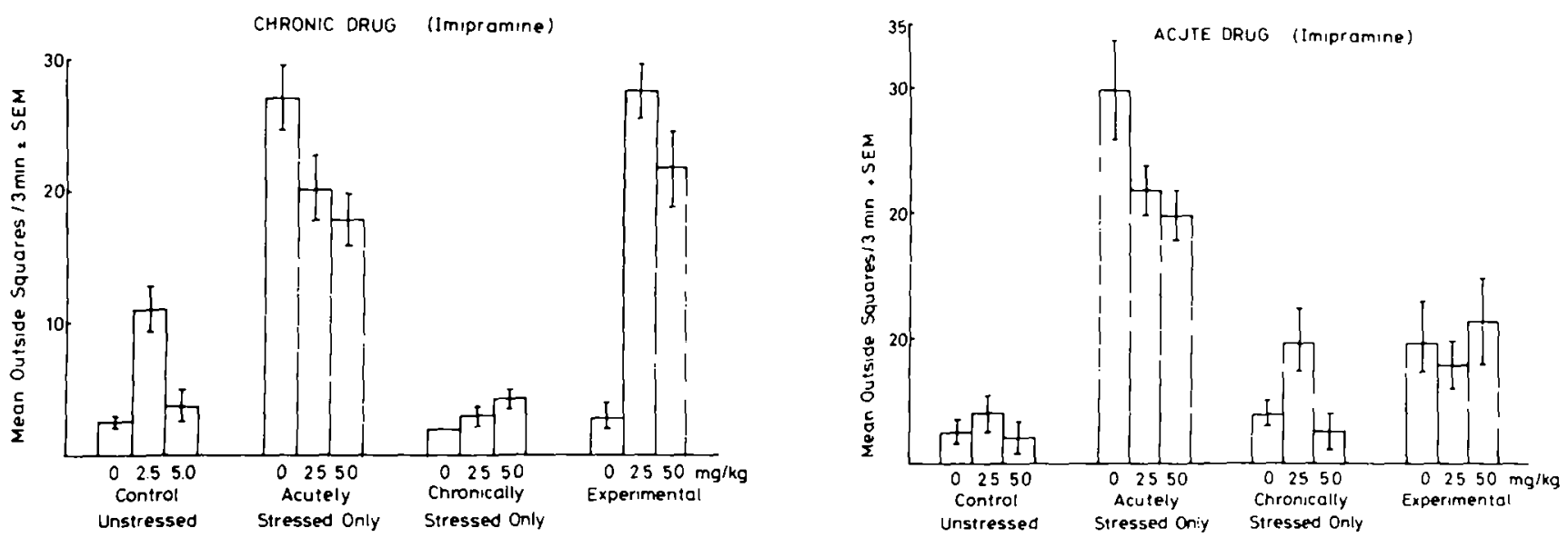

FIG. 1. Effects of acute and chronic stress and drug administration upon open field motor activity in the rat (mean outside squares/3 $\min \pm$ standard error). A single placement is used and a factorial experimental design is employed. Right-hand side of figure displays acute ( 1 hr) drug administration effects and left-hand side of figure displays chronic ( 3 week) drug administration effects.
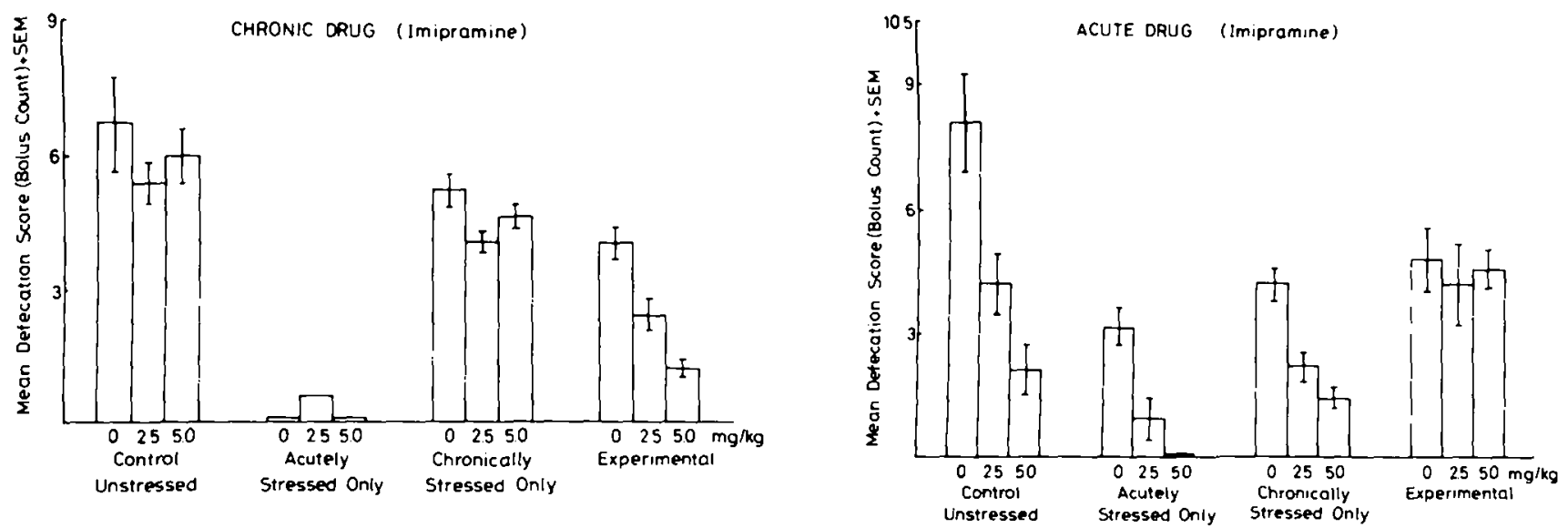

FIG. 2. Effects of acute and chronic stress and drug administration upon open field defecation in the rat (mean bolus count/per session \pm standard error). A single placement is used and a factorial experimental design is employed. Right-hand side of figure displays acute ( 1 hr) drug administration effects and left-hand side of figure displays chronic ( 3 week) drug administration effects.

period corresponding to the chronic stress period; and (4) drug dose, rats were exposed to vehicle $(0.9 \%$ sodium chloride) or 2.5 or $5.0 \mathrm{mg} / \mathrm{kg}$ of imipramine $\mathrm{HCl}, 1 \mathrm{ml} / \mathrm{kg}$ intraperitoneally. All drugs were prepared within $72 \mathrm{hr}$ of use.

Four behavioral measures were examined. All measures have been described in detail in previous reports $[2,3]$. Initial motor activity in the periphery of the field (min $0-3$ ) was designated outside square activity. A bolus count at the close of testing was designated defecation score. Latencies (in sec) to leave initial home square, and for defecation were also collected and analyzed.

\section{Statistical Analysis}

All data are presented as means and standard errors. Analysis was by 4 -factor analysis of variance based upon the above factors. In the interests of brevity interaction effects are reported only if they reach a level of statistical significance of $p<0.05$.

\section{RESLLTS}

Overall, the behaviorally activating effects of acute stress were demonstrated, as was their reduction by chronic stress and reinstatement by chronic drug treatment. Acute drug treatment proved ineffective. Drug dose also affected outcome. Findings were confirmed statistically based upon main and interaction effects as reported below. Results are presented graphically in Figs. 1-4.

The above pattern of results may be seen for the initial activity scores which are presented in Fig. 1. Activity was significantly increased following acute stress, and this was blunted by a history of prior stress. Chronic, but not acute, drug treatment restored the activating effects of acute stress. Main effects for drug chronicity, acute stress, and chronic stress were significant, $F^{\prime} s(1,15)=18.1,15.8,118.6$ in all cases, $p<0.001$; however, a main effect of drug dose was not, $F(2,15)=1.8$, n.s. In further support of the hypothesized interactions chronic stress affected responding to acute stress, $F(1,15)=42.2, p<0.001$, and this in turn interacted 

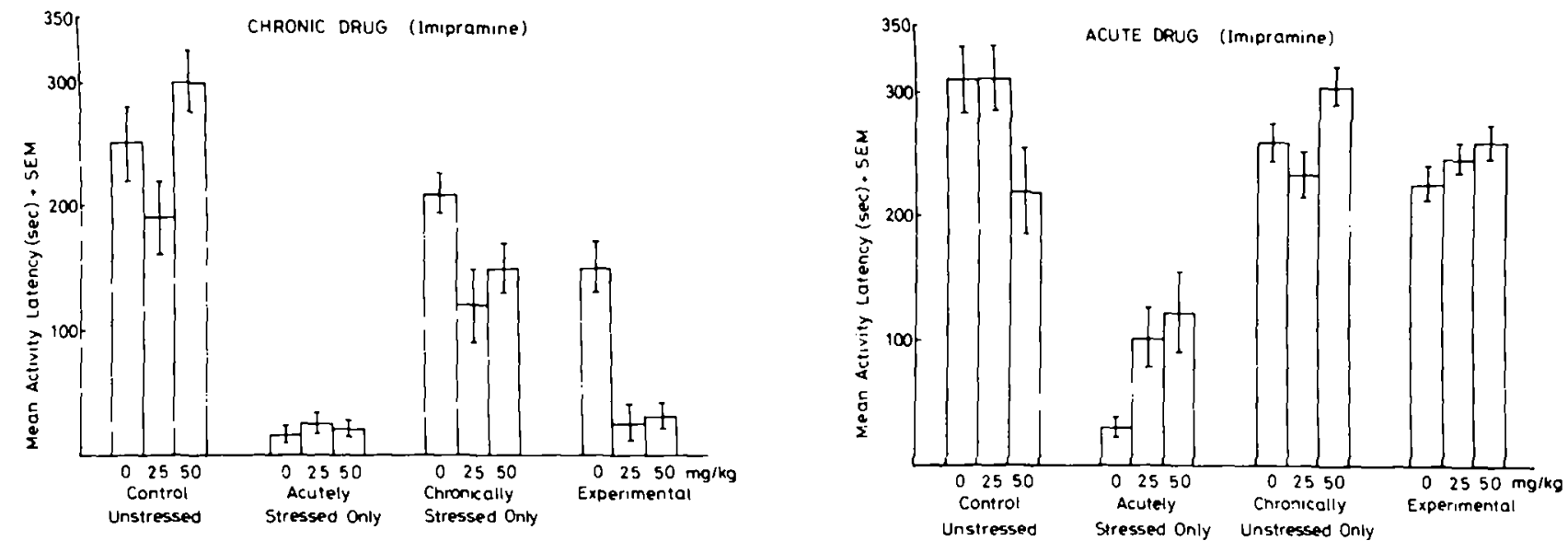

FIG. 3. Effects of acute and chronic stress and drug administration upon open field activity in the rat. Mean latency scores \pm S.E.M. (in sec) are presented, and a factorial experimental design is employed. Right-hand side of figure displays acute ( 1 hr) drug administration effects and left-hand side of figure displays chronic ( 3 week) drug administration effects.
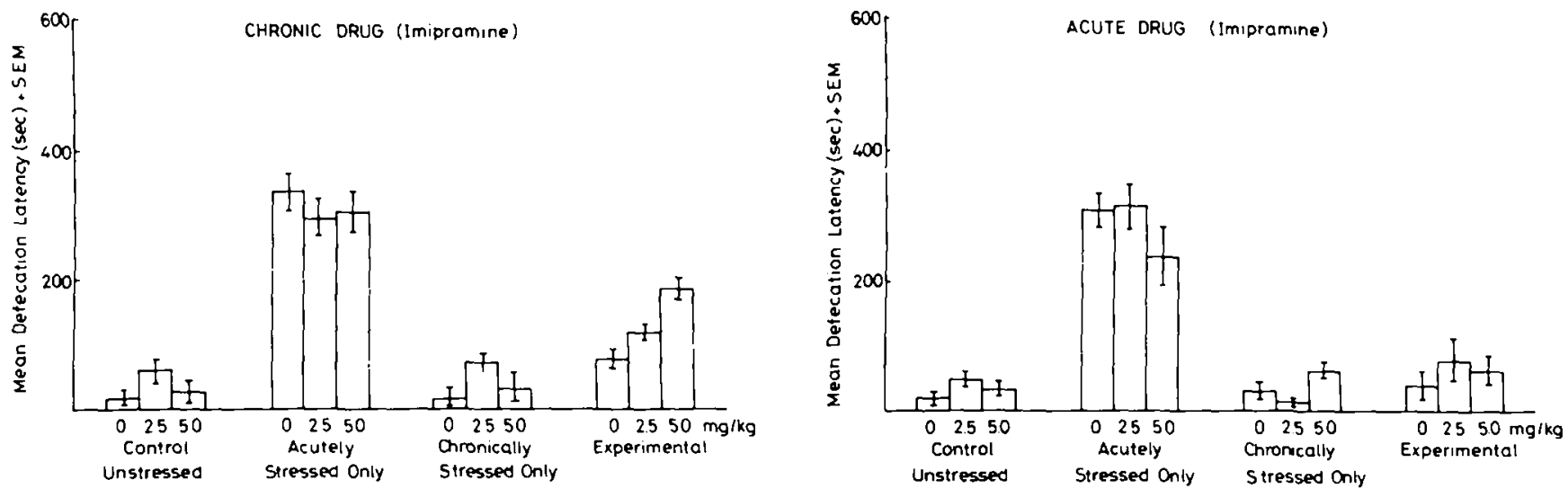

FIG. 4. Effects of acute and chronic stress and drug administration upon open field defecation (mean latency +S.E.M. (sec)). A single placement is used and a factorial experimental design is employed. Right-hand side of figure displays acute (1 hr) drug administration effects and left-hand side of figure displays chronic ( 3 week) drug administration effects.

with drug dosage, $\mathrm{F}(2,15)=12.3, p<0.0001$. Other significant interactions included acute stress by drug dose, $F(2,15)=3.9$, $p<0.05$, and the three way interactions of drug chronicity and dose with acute stress, $F(2,15)=4.6, p<0.05$, and chronic stress, $\mathrm{F}(2,15)=4.0, p<0.05$.

Effects of treatments and their combinations upon the defecation measure are presented in Fig. 2. Main effects for drug chronicity, acute stress, chronic stress, and drug dosage level were significant, $F_{s}=4.6,6.9,149.9,14.4, d f s$ as above, all $p^{\prime} s<0.05$ ). An interaction of acute and chronic stress again was present, $\mathrm{F}(1,15)=7.4, p<0.001$, as was the interaction of acute and chronic stress with drug dose, $F(2,15)=8.0$, while other interaction effects were not significant.

Effects upon motor latencies are presented in the third figure. Main effects for drug chronicity and chronic stress were significant, $\mathrm{F}=3.8,26.3, d f$ as above, $p<0.05$, while main effects for acute stress and drug dosage were not, $F^{\prime} s=0.3,1.3, d f$ as above, $p>0.05$. A significant interaction of acute and chronic stress was found, $F(1,15)=13.7$, $p<0.001$, and this was dependent upon chronic drug administration, $\mathrm{F}=6.4, p<0.05$. Other effects include drug chronicity with acute stress, $F(1,15=4.5, p<0.05$, and drug dose with acute stress, $\mathrm{F}(2,05)=4.1, p<0.05$, and the 3 way interaction of drug chronicity, stress chronicity and drug dose, $\mathrm{F}(2,15)=4.7, p<0.05$.

Effects upon defecation latencies are presented in the final figure (Fig. 4). Main effects of acute stress and chronic stress were present, $F^{\prime} s=57.8,137.4$, df as above, $p<0.00001$, while main effects for drug chronicity and level were not, $F$ 's $=0.5,0.3$. An interaction of acute and chronic stress was present, $F=55.5$, as were significant influences of drug dosage and chronicity upon the above, $F=4.1,5.0$.

\section{DISCUSSION}

The above analysis routinely confirmed the influences of chronic drug administration and chronic stress upon the depression model, as well as the normal interaction of acute and chronic stress, and higher order interactions of both 
stress factors with chronic drug treatment. Acute stress was a significant factor in most analyses, with drug dosage by itself generally playing a lesser role. Indeed, dosage per se did not significantly affect behavior in most cases. This might be expected on purely statistical grounds given the predominance of control groups which were not affected by drug treatment. However, dose did interact with chronic stress, or with the combined effects of chronic and acute stress, as might be predicted. Of the 4 measures, in only one case (defecation latency, see Fig. 3) and in that case to a rather limited degree, was an acute antidepressant effect observed. Careful examination of this finding points to several possible explanations. It might be noted, for example, that the unstressed latency baseline was increasing at this point. thus, the change may not be stress-specific. In addition, chronic treatment is significantly superior to acute treatment. The overall success of chronic drug effects upon all measures must again be emphasized. The present findings demonstrate that treatment regimens effective in the stress model may resemble clinical treatments upon such critical variables as time course and dose. This in turn. suggests the chronic stress model bears several similarities to depression.

In the present report four measures were taken. If an analogy is to be drawn with clinical findings then it should be kept in mind that monotonic dose-response relationships are more difficult to demonstrate in clinical research settings than are nominal relationships between treatment and improvement beyond some criterion. Thus it might be argued that at a level of face validity a binary measure evaluated non-parametrically, or some measure sensitive to a nonmonotonic course of improvement, is most appropriate. Of the major behavioral measures the activity measure most directly demonstrates a "therapeutic window" involving non-monotonic improvement. Thus of the broadly defined categories of activity and defecation the former may be more representative of a clinical picture. It should be noted that for the rat these measures often vary inversely under normal circumstances of first exposure. The nonlinear trend in activity is therefore of particular interest.
The present interpretation presupposes that imipramine given chronically is not itself debilitating. One side effect of chronic imipramine, for example, is a relative reduction in normal weight gain. Factors such as anorexia. illness, and debilitation are best controlled for by the use of active control substances (i.e., nonantidepressants with similar profiles of side effects). In this regard it might be noted that scopolamine, a potent anticholinergic; amphetamine, a catecholamine releaser; and other psychotropic compounds. e.g., tripellinamine. oxazepam. and haloperidol have been evaluated in the stress model. Related doses and identical time courses have been employed, and in no case has a similar behavioral profile been observed. On the other hand chronic electroconvulsive shock and monoamine oxidase inhibitors, which are clinically effective, but quite different in their side effects in comparison with imipramine appear similar to the latter in their effects upon stress and open field behavior. Thus, there is a high degree of convergence between clinical activity and preclinical response in the stress model.

Finally it should be noted that pharmacokinetic factors, which are critical at a clinical level, have not been investigated in the model. In this regard, it might be noted that a $24-48 \mathrm{hr}$ washout period preceded testing. Nonetheless, actual serum drug levels are a more valid indicator, and ideally this factor should also be taken into account in any interpretation of drug efficacy. This points to an area of potential future investigation.

\section{ACKNOWLEDGEMEN]}

Supported by grant 31588 from the National Institute of Mental Health to Dr. B. J. Carroll. Dr. Katz is currently an Alfred P. Sloan foundation fellow in Neuroscience. The editorial assistance of Anne Marie Skepenaitis, technical assistance of Mike Sibel, and statistical assistance of $R$. C. Shea are acknowledged with gratitude. Imipramine was generously donated by Ciba-Geigy.

\section{REFERENCES}

1. Dixon, W. J. and F. Massey. Introduction to Statistical Analysis. New York: McGraw-Hill, 1969.

2. Katz, R. J. Animal model of depression-effects of electroconvulsive shock therapy. Ne'urosci. Biohehar. Rev. 5: 273-277, 1981.

3. Katz, R. J. Amitriptyline and scopolamine in an animal model of depression. Neurosci. Biobehav. Rev. 5: 265-271, 1981.
4. Oswald, I., V. Brezinova and D. L. F. Dunleavy. On the slowness of action of tricyclic antidepressant drugs. Br. J. Psychiat. 120: 673-677. 1972.

5. Roth, K. A. and R. J. Katz. Further studies on a novel animal model of depression therapeutic effects of a tricyclic antidepressant. Neurosci. Biobchav. Rev. 5: 253-258, 1981.

6. Risch, S. C., L. Y. Huey and D. S. Janowsky. Plasma levels of tricyclic antidepressants and clinical efficacy: Review of the literature, Part 1.J. clin. Psychiat. 40: 9-24. 1979. 